\title{
Focused section on robust perception and learning for robots in dynamic environments
}

\author{
Huaping $\mathrm{Liu}^{1} \cdot$ Xiaoli Bai $^{2} \cdot$ Jun Ueda ${ }^{3} \cdot$ Ye Zhao ${ }^{3}$
}

Published online: 2 November 2019

(c) Springer Nature Singapore Pte Ltd. 2019

Intelligent robots can adapt to dynamic and unstructured environments when perception and learning are seamlessly coupled. To break through the bottleneck that limits robot intelligence, it is important to solve the issues of robust perception and learning. Key features of robust perception and learning include multi-modal fusion, active perception, cross-modal learning, lifelong learning and adversarial learning. The primary objectives of this focused section are dedicated to soliciting the state-of-the-art and novel contributions addressing fundamental challenges and practical issues in all aspects of robust perception and learning.

In this special issue, we mainly focus on robust perception, control and learning, addressing both original algorithmic development and new applications. The accepted contributions can be divided into the following three parts:

The first part contains two survey papers. In the first survey Coverage Optimization of Visual Sensor Networks for Observing 3-D objects: Survey and Comparison, the authors provide detailed comparison of mainstream coverage optimization methods for visual sensor networks, including the greedy algorithm, genetic algorithm, particle swarm optimization, binary integer programming and differential evolution algorithm. In the second one, Survey of Imitation Learning for Robotic Manipulation, the authors provide the survey of imitation learning of robotic manipulation and explore

Huaping Liu

hpliu@tsinghua.edu.cn

Xiaoli Bai

xb25@soe.rutgers.edu

Jun Ueda

jun.ueda@me.gatech.edu

Ye Zhao

ye.zhao@me.gatech.edu

1 Tsinghua University, Beijing, China

2 Rutgers University, New Brunswick, USA

3 Georgia Institute of Technology, Atlanta, USA the future research trend, from the aspects of demonstration, representation and learning.

The second part contains four papers which focus on the intelligent planning and control. In the paper A Bilevel Optimal Motion Planning (BOMP) Model with Application to Autonomous Parking, the authors treats motion planning as an optimal control problem, in which the upper level is designed for vehicle nonlinear dynamics, and the lower level is for geometry collision-free constraints. In Research on Attack Angle Tracking of High Speed Vehicle based on PID and FLNN Neural Network, the authors addressed the problem of a kind of pitch channel dynamic model of hypersonic aircraft considered with both the model of engine and the model of elastic shape. In A Distributed Approach for Road Clearance with Multi-Robot in Urban Search and Rescue Environment, the authors utilize the RoboCup rescue simulation for disaster management where heterogeneous field agents (fire-brigade agent, ambulance agent, and police force agent) collaborate to manage a mimicked calamity situation. In Universal Output Feedback and Nussbaum Gain Adaptive Control of Supersonic Missiles with Unknown Control Direction, the authors develop two kinds of novel universal output feedback controllers to solve the unmeasurable states problem for a uncertain missile system.

The third part contains four papers which focus on the intelligent planning and control. In Machine-Learning-based Hand Motion Recognition System by Measuring Forearm Deformation with a Distance Sensor Array, the authors focus on forearm deformation which is an alternative source of information for hand motion recognition. In Object Detection Based on Colour and Shape Features for Service Robot in Semi-Structured Indoor Environment, the authors developed a new visual object detection method based on color and shape features. In Structural Constraint Deep Matrix Factorization for Sequential Data Clustering, the authors propose structural constraint deep matrix factorization which captures the ordered structure information into the deep matrix factorization process to improve data representation. 
In A Computer Vision and Artificial Intelligence based Cost-Effective Object Sensing Robot, the authors present an enhanced object detection system incorporating a few wellknown computer vision techniques, machine learning algorithms, as well as smart sensors in a more organized way. In Spatial Attention Model Based Target Detection for Aerial Robotic Systems, the authors develop a novel target detection method based on spatial attention model, which changes the existing methods to enhance the features of target areas by enhancing global semantic information.

We are glad to see that the papers included in this special issue cover different aspects of theoretical algorithm, technology and applications. During the review process, reviewers contributed a lot to the quality of this special issue by thoroughly evaluating the papers and providing a great deal of constructive comments. Last, but not the least, the completion of this special issue cannot be separated from the strong support from the Editor-in-Chief, Prof. Kok-Meng Lee. We would like to thank him for his great support.

Publisher's Note Springer Nature remains neutral with regard to jurisdictional claims in published maps and institutional affiliations.

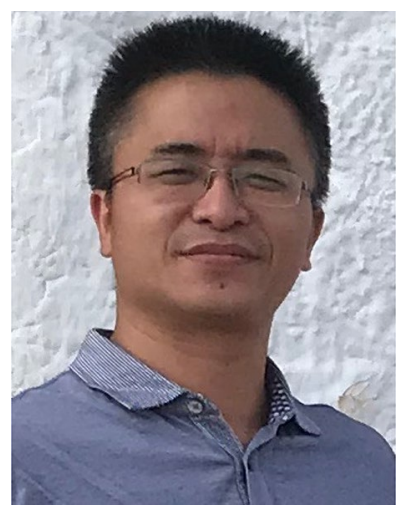

Huaping Liu received the Ph.D. degree from Tsinghua University, Beijing, China. He is currently an Associate Professor with the Department of Computer Science and Technology, Tsinghua University, Beijing, China. His research interests include robot perception and learning. Dr. Liu served as a Senior Program Committee Member for the International Joint Conference on Artificial Intelligence 2018. He was a recipient of the Andy Chi Best Paper Award in 2017. He served as the Area Chair for Robotics Science and Systems 2018. He is an Associate Editor for some journals, including the IEEE TRANSACTIONS ON AUTOMATION SCIENCE AND ENGINEERING, IEEE TRANSACTIONS ON INDUSTRIAL INFORMATICS, IEEE ROBOTICS AND AUTOMATION LETTERS, Neurocomputing, and Cognitive Computation, and some conferences, including the International Conference on Robotics and Automation and the IEEE/RSJ International Conference on Intelligent Robots and Systems.

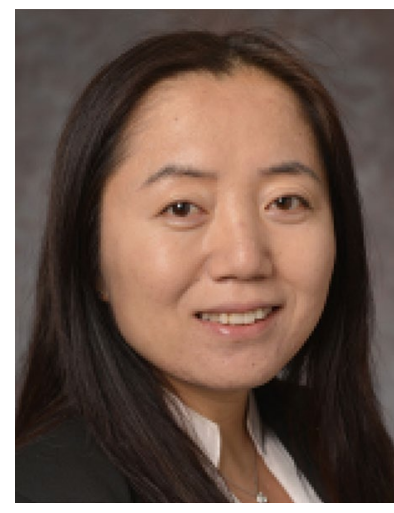

Xiaoli Bai is an Assistant Professor in the department of Mechanical and Aerospace Engineering at Rutgers, The State University of New Jersey. She obtained her $\mathrm{PhD}$ degree of Aerospace Engineering from Texas A\&M University. Her current research interests include spacecraft guidance, control, and space robotics; astrodynamics; and Unmanned Aerial Vehicle navigation and control. She was a recipient of The 2019 NASA Early Career Faculty award, The 2016 Air Force Office of Scientific Research Young Investigator Research Program award, Outstanding Young Aerospace Engineer Award from Texas A\&M University in 2018, A. Water Tyson Assistant Professor Award from Rutgers in 2018, and Amelia Earhart Fellowship.



Jun Ueda received the B.S., M.S., and $\mathrm{Ph} . \mathrm{D}$. degrees from Kyoto University, Kyoto, Japan, in 1994, 1996, and 2002, respectively, all in mechanical engineering. He joined the G.W. Woodruff School of Mechanical Engineering, Georgia Institute of Technology as an Assistant Professor in 2008 and is currently an Associate Professor and Woodruff Faculty Fellow. From 1996 to 2000, he was a Research Engineer with the Advanced Technology Research and Development Center, Mitsubishi Electric Corporation,

Japan. He was an Assistant Professor with Nara Institute of Science and Technology, Japan, from 2002 to 2008. During 2005-2008, he was a visiting scholar and lecturer with the Department of Mechanical Engineering, Massachusetts Institute of Technology. He was the Director for the Robotics Ph.D. Program with Georgia Tech for 2015-2017. Dr. Ueda was the recipient of the Fanuc FA Robot Foundation Best Paper Award in 2005, the IEEE Robotics and Automation Society Early Academic Career Award in 2009, and the Advanced Robotics Best Paper Award in 2015. He is currently an Associate Editor for IEEE RAS ROBOTICS AND AUTOMATION LETTERS, International Journal of Intelligent Robotics and Applications, and IEEE TRANSACTIONS ON ROBOTICS. He is the author of Cellular Actuators: Modularity and Variability in MuscleInspired Actuation (Butterworth-Heinemann, 2017), and Human Modeling for Bio-Inspired Robotics (Academic Press, 2017). 


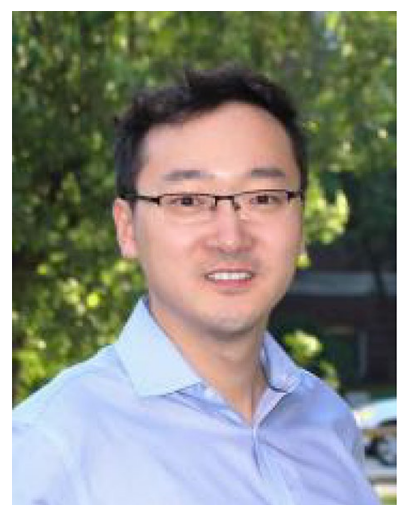

Ye Zhao is an Assistant Professor at The George W. Woodruff School of Mechanical Engineering, Georgia Institute of Technology. He leads the Laboratory for Intelligent Decision and Autonomous Robots at Georgia Tech. He is affiliated with the Institute for Robotics and Intelligent Machines (IRIM) and Machine Learning Center. He was a Postdoctoral Fellow at SEAS, Harvard University. He received his Ph.D. degree in Mechanical Engineering from The University of Texas at Aus-

tin in 2016. His research interests lie broadly in planning, control, decision-making, and optimization algorithms of highly dynamic, contact-rich, and human-centered robots. He is especially interested in challenging locomotion and manipulation problems with formal guarantees on robustness, agility, real-time, and autonomy. Dr. Zhao's coauthored work has been recognized as the 2017 Thomson Reuters Highly Cited Paper and 2016 IEEE-RAS best whole-body control paper and video award finalist. He serves as an Associate Editor of IEEERAS Robotics and Automation Letters. He was a Co-Chair of the IEEE Robotics and Automation Society (RAS) Student Activities Committee and a Committee Member of IEEE-RAS Member Activities Board. He is also an ICT Chair of the 2018 IEEE/RSJ International Conference on Intelligent Robots and Systems, and serves as an Associate Editor of IROS and Humanoids Conferences. 